\title{
Interactions of nanodiamonds and surfactants in aqueous suspensions
}

\author{
A. M. Vervald ${ }^{1}$, S. A. Burikov ${ }^{1}$, I. I. Vlasov ${ }^{2}$, O. A. Shenderova ${ }^{3}$, T. A. Dolenko ${ }^{1}$ \\ ${ }^{1}$ Physical Department, M. V. Lomonosov Moscow State University, Moscow, Russia \\ ${ }^{2}$ A. M. Prokhorov General Physics Institute, Russian Academy of Sciences, Moscow, Russia \\ ${ }^{3}$ Adamas Nanotechnologies, Inc., Raleigh, NC, USA \\ alexey.vervald@physics.msu.ru
}

PACS 61.46.-w, 82.30.Rs, 82.70.Uv

DOI 10.17586/2220-8054-2018-9-1-49-51

\begin{abstract}
In this study, with the use of laser Raman spectroscopy the significant difference in intermolecular interactions of detonation nanodiamonds with hydrophilic and hydrophobic surface groups in aqueous solution of surfactants was observed. It was found that at low concentrations of sodium octanoate (before the micelle formation) the weakening of hydrogen bonds by nanodiamonds has a different dynamics for hydrophobic and hydrophilic nanodiamonds. However, with the addition of surfactants, this effect gradually decreases for both types of nanodiamonds and ends after the formation of micelles. Such effects are explained by the "shielding" effect of surfactant molecules surrounding nanodiamond particles.
\end{abstract}

Keywords: nanodiamonds, surfactants, suspensions, hydrogen bonds, dispersibility.

Received: 20 June 2017

Revised: 11 September 2017

\section{Introduction}

One of the features for nanodiamonds (NDs) is their complex chemically active surface, due to which NDs can be widely used in biomedicine as drugs carriers, adsorbents, or as the basis for various bioconjugates [1,2]. Alternatively, the chemical activity of the surface groups leads to the formation of ND's aggregates [3]. To improve the NDs' dispersibility, surfactants are often added in suspensions [4]. However, physical picture of the interactions between these nanoparticles and molecules of surfactants is not fully understood.

It is known that in suspensions, surfactants [5], as well as NDs [3,6,7], substantially change the structure of solvent and, primarily, the strength of hydrogen bonds. The authors [6,7] showed that in aqueous suspensions the detonation nanodiamonds (DNDs) weaken the hydrogen bonds in bulk water and such changes depend on the type of ND's surface groups.

In this study, we investigated the interactions of hydrophilic (DND-COOH) and hydrophobic (DND-H) surface groups of DNDs with the surfactant sodium octanoate in water using dynamic light scattering and Raman spectroscopy techniques.

\section{Experimental part}

The functionalization and preparation of the initial aqueous suspensions of DND-COOH (10 g/L) and of DNDH (5.3 g/L) was carried out by Adámas Nanotechnologies, Inc. (USA) and in Diamond Sensors Laboratory, CEA (France) [7], accordingly. As a surfactant, sodium octanoate $\left(\mathrm{C}_{8} \mathrm{H}_{15} \mathrm{NaO}_{2}\right.$, hereinafter $\left.\mathrm{NaC} 8\right)$ of the pure ph. Eur class (AppliChem) was used. The critical micelle concentration (CMC) of $\mathrm{NaC} 8$ at which spherical micelles are formed is around $330-370 \mathrm{mM}$ [8]. From the initial suspensions, two aqueous suspensions with concentration $0.5 \mathrm{~g} / \mathrm{L}$ for DND-COOH and $0.25 \mathrm{~g} / \mathrm{L}$ for DND-H were prepared. During the experiment, NaC8 was added in the water and DNDs suspensions so that the concentration of $\mathrm{NaC} 8$ in these three samples was changed in the range of $0-1700 \mathrm{mM}$ with the step $20-100 \mathrm{mM}$.

The sizes of DNDs in the suspensions were measured by dynamic light scattering (DLS) using device ALVCGS 5000/6010 (Germany). The size of DND-COOH in aqueous suspension was $12 \mathrm{~nm}$, the size of DND-H was $180 \mathrm{~nm}$. With the addition of $200 \mathrm{mM}$ of NaC8 the second fraction of DND-COOH with size $70 \mathrm{~nm}(20 \%$ of the total number of particles) was formed. With the addition of $200 \mathrm{mM}$ of $\mathrm{NaC} 8$ the DND-H aggregates broke up into two fractions: $60 \%$ of the total number with the size $24 \mathrm{~nm}, 40 \%$ - with the size $71 \mathrm{~nm}$.

Raman spectra of the prepared solutions and suspensions were obtained with argon laser excitation $(488 \mathrm{~nm}$, $400 \mathrm{~mW}$ ) from $400-4500 \mathrm{~cm}^{-1}$ [6] (Fig. 1a). From $3000-3900 \mathrm{~cm}^{-1}$, the band of $\mathrm{OH}$ stretching vibrations is located, while the bands for CHx stretching vibrations of different symmetries were found from $2820-3000 \mathrm{~cm}^{-1}$. The fluorescent pedestal was approximated by the 2 nd degree polynomial. 

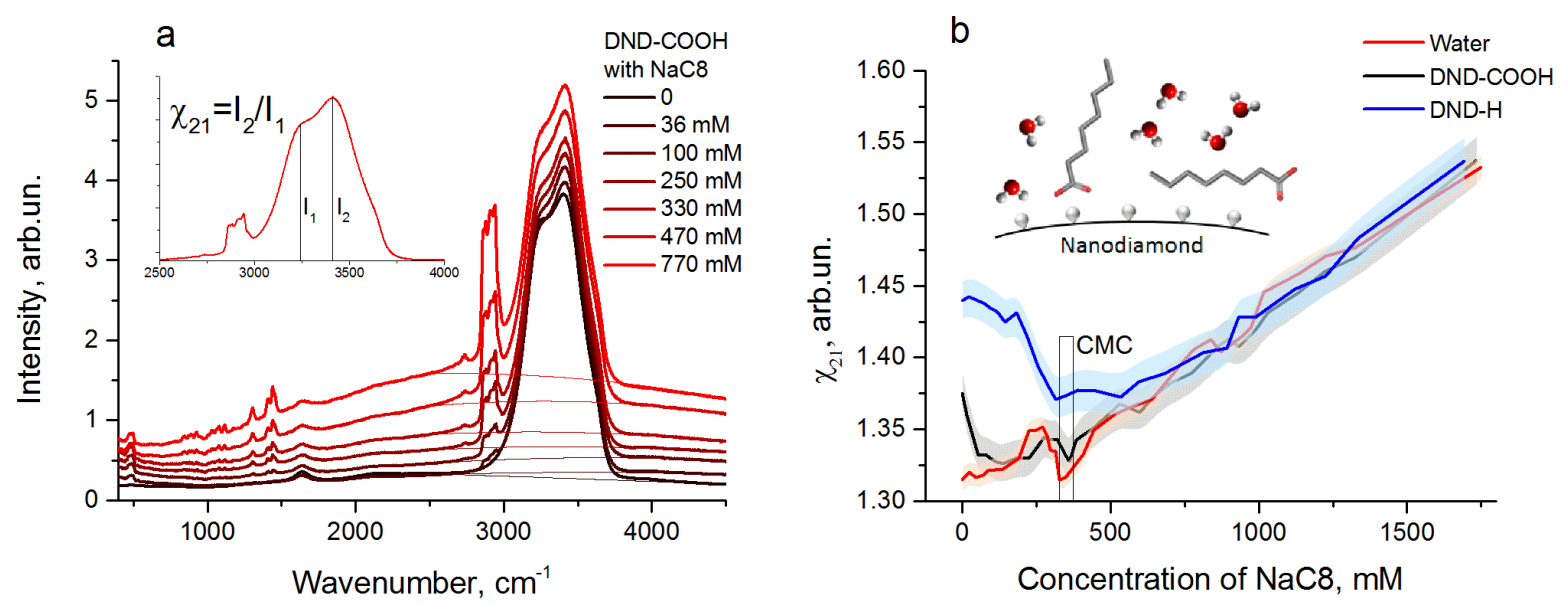

FIG. 1. (a) Raman spectra of aqueous suspension of DND-COOH $(0.5 \mathrm{~g} / \mathrm{L})$ with $\mathrm{NaC} 8$ at various concentrations; (b) Dependencies of the parameter $\chi_{21}$ on the $\mathrm{NaC} 8$ concentration in three examined suspensions; in the inset, there is illustration of probable interactions of surfactant molecules with the surface of DND-H in water

\section{Results and discussion}

The stretching band of $\mathrm{OH}$ groups (Fig. 1a) is very sensitive to the changes in the hydrogen bond strength. Low-frequency range of the stretching band ( $\mathrm{I}_{1}$ region) is caused by the vibrations of $\mathrm{OH}$ groups with strong hydrogen bonds, high-frequency range ( $\mathrm{I}_{2}$ region) is caused by the vibrations of $\mathrm{OH}$ groups with weak hydrogen bonds. Thus, the parameter $\chi_{21}$ equals the ratio of intensity $\mathrm{I}_{1}$ to intensity $\mathrm{I}_{2}$ (Fig. 1a, inset), which characterizes the strength of hydrogen bonds. When the parameter $\chi_{21}$ increases, the hydrogen bonds are weakened, when the parameter $\chi_{21}$ decreases they are strengthened. In Fig. 1b, the dependencies of the parameter $\chi_{21}$ on concentration $C$ of sodium octanoate in water and DNDs suspensions are shown. In these dependencies, two parts can be clearly distinguished: the complex behavior of the $\chi_{21}(C)$ at low concentrations of $\mathrm{NaC} 8$ and the monotonic growth of the $\chi_{21}(C)$ at $C>\mathrm{CMC}$.

In an aqueous solution of $\mathrm{NaC} 8$ with the increase of $\mathrm{NaC} 8$ concentration up to the $\mathrm{CMC}$, the hydrogen bonds are significantly weakened. At the beginning of micelle formation (in CMC region), the hydrogen bonds are sharply strengthened (the parameter $\chi_{21}$ sharply decreases) due to water extrusion and compression by the forming micelles [5]. After micelle formation $(C>\mathrm{CMC})$, with the addition of $\mathrm{NaC} 8$ in the suspension, the monotonic weakening of the hydrogen bonds is observed.

The presence of hydrophilic and hydrophobic DNDs in water weakens the hydrogen bonds [6,7]. With the addition of $\mathrm{NaC} 8$, the aggregation of DND-COOH (see results of DLS), or the decrease of the DND-COOH total surface, takes place. As a result, the influence of DND-COOH on the hydrogen bonds decreases: $\chi_{21}$ for the DND-COOH suspension tends to that for the aqueous solution of $\mathrm{NaC} 8$ at $C=250 \mathrm{mM}$ (Fig. 1b). With further addition of $\mathrm{NaC} 8$ in the $\mathrm{DND}-\mathrm{COOH}$ suspension, the dynamic of hydrogen bonds repeats the dynamic of those in the $\mathrm{NaC} 8$ aqueous suspension.

The dynamic of hydrogen bonds in the DND-H suspension in the presence of $\mathrm{NaC} 8$ differs from that in the DND-COOH suspension significantly. Since DND-H disaggregates with the addition of NaC8 (see results of DLS), it would be expected that the hydrogen bonds would be further weakened. However, at $C<\mathrm{CMC}$ the $\chi_{21}$ for the DND-H gradually decreases up to CMC without any wavering, and then it behaves like the parameter $\chi_{21}(C)$ for other samples. We explain this by the gradual "shielding" of the surface of DND-H from water by the molecules of $\mathrm{NaC} 8$ (the inset in Fig. 1b). Due to the hydrophobic positively charged surface of the DND-H, the molecules of $\mathrm{NaC} 8$ can be attracted to it by their head (Coulomb interaction) or tail (proximity of the hydrophobic areas). At $C>\mathrm{CMC}$ the second method become favorable and that results in the further similarity of the dependencies $\chi_{21}(C)$.

Thus, using Raman spectroscopy the significant difference in the intermolecular interactions of hydrophobic and hydrophilic DNDs in the aqueous solutions of surfactants was found. For suspensions of DND-H, the partial "shielding" of DND-H by the surfactant molecules from the interactions with the solvent and the subsequent complete "shielding" under micelle formation were observed. Such complex interactions between nanodiamonds and surrounding molecules should be considered when using nanodiamonds in biomedicine. 


\section{Acknowledgements}

This study has been performed with support from the grant of Russian Science Foundation (project No. 17 12-01481). The authors express their deep appreciation to Petit T., Girard H. A. and Arnault J.-Ch. for DND-H suspensions samples.

\section{References}

[1] Turcheniuk K., Mochalin V.N. Biomedical Applications of Nanodiamond (Review). Nanotechnology, 2017, 28, P. 252001-252027.

[2] Rosenholm J.M., Vlasov I.I., et al. Nanodiamond-Based Composite Structures for Biomedical Imaging and Drug Delivery (Review). J. Nanosci. Nanotechnol., 2015, 15, P. 959-971.

[3] Batsanov S.S., Lesnikov E.V., DanKin D.A., Balakhanov D.M. Water shells of diamond nanoparticles in colloidal solutions. Appl. Phys. Lett., 2014, 104, 133105.

[4] Neugart F., Zappe A., et al. Dynamics of diamond nanoparticles in solution and cells. Nano Lett., 2007,7 (12), P. 3588-3591.

[5] Dolenko T.A., Burikov S.A., et al. Raman Spectroscopy of Micellization-Induced Liquid-Liquid Fluctuations in Sodium Dodecyl Sulfate Aqueous Solutions. J. Mol. Liq., 2015, 204, P. 44-49.

[6] Vervald A.M., Burikov S.A., et al. Relationship Between Fluorescent and Vibronic Properties of Detonation Nanodiamonds and Strength of Hydrogen Bonds in Suspensions. J. Phys. Chem. C, 2016, 120 (34), P. 19375-19383.

[7] Petit T., Puskar L., et al. Unusual Water Hydrogen Bond Network around Hydrogenated Nanodiamonds. J. Phys. Chem. C, 2017, 121, P. 5185-5194.

[8] Rosenholm J.B. The structure and properties of medium-chain surfactant solutions: a case study of sodium octanoate. Adv. Colloid Interface Sci., 1992, 41 (1), P. 197-239. 